\title{
TLR-2 and TLR-9 are sensors of apoptosis in a mouse model of doxorubicin-induced acute inflammation
}

\author{
DV Krysko ${ }^{1,2,6}$, A Kaczmarek ${ }^{1,2,6}$, O Krysko ${ }^{3}$, L Heyndrickx ${ }^{1,2}$, J Woznicki ${ }^{1,2}$, P Bogaert $^{1,2}$, A Cauwels ${ }^{1,2}$, N Takahashi ${ }^{1,2}$, S Magez $^{4,5}$, \\ C Bachert ${ }^{3}$ and $P$ Vandenabeele ${ }^{*, 1,2}$
}

\begin{abstract}
Anthracycline antibiotics are inducers of an immunogenic form of apoptosis that has immunostimulatory properties because of the release of damage-associated molecular patterns. To study the mechanisms used by the innate immune system to sense this immunogenic form of cell death, we established an in vivo model of cell death induced by intraperitoneal injection of doxorubicin, a prototype of anthracyclines. The acute sterile inflammation in this model is characterized by rapid influx of neutrophils and increased levels of IL- 6 and monocyte chemotactic protein-1. We demonstrate that acute inflammation induced by doxorubicin is associated with apoptosis of monocytes/macrophages and that it is specific for doxorubicin, an immunogenic chemotherapeutic. Further, the inflammatory response is significantly reduced in mice deficient in myeloid differentiation primary response gene 88 (MyD88), TLR-2 or TLR-9. Importantly, a TLR-9 antagonist reduces the recruitment of neutrophils induced by doxorubicin. By contrast, the acute inflammatory response is not affected in TRIF ${ }^{\text {Lps2 }}$ mutant mice and in TLR-3, TLR-4 and caspase-1 knockout mice, which shows that the inflammasome does not have a major role in doxorubicin-induced acute inflammation. Our findings provide important new insights into how the innate immune system senses immunogenic apoptotic cells and clearly demonstrate that the TLR-2/TLR-9-MyD88 signaling pathways have a central role in initiating the acute inflammatory response to this immunogenic form of apoptosis.
\end{abstract}

Cell Death and Differentiation (2011) 18, 1316-1325; doi:10.1038/cdd.2011.4; published online 11 February 2011

Apoptotic cell death is a tightly regulated physiological form of cell death, and one of its important aspects is targeted elimination of apoptotic cells without induction of inflammation or tissue scarring. This process serves as an integral part of homeostatic mechanisms. Apoptotic cells exert antiinflammatory effects by triggering the release of transforming growth factor beta (TGF- $\beta$ ), interleukin (IL)-10, plateletactivating factor and prostaglandin E2 from engulfing cells. ${ }^{1,2}$ In addition, it has been shown that direct contacts between apoptotic cells and phagocytes contribute to the immunosuppressive effect of apoptotic cells. ${ }^{3,4}$ Apoptotic cells are cleared via a 'zipper'-like mechanism of internalization. ${ }^{5}$ In vivo, apoptotic cells are rapidly sensed and cleared not by neutrophils but preferentially by monocytes, which are attracted to the site of cell death by the release of chemoattractants. Elliott et al. ${ }^{6}$ reported that supernatant obtained from apoptotic Jurkat cells, killed by UV or anti-Fas, induces recruitment of monocytes in a $\mathrm{P}_{2} \mathrm{Y}_{2}$-dependent manner by releasing nucleotides such as adenosine triphosphate (ATP) and uridine triphosphate (UTP). In fact, apoptotic cells generate negative signals that could prevent neutrophil recruitment and dampen responsiveness of the innate immune system. Recently, it was shown that apoptotic Burkitt lymphoma cells specifically inhibit neutrophil chemotaxis by secreting lactoferrin, a pleiotropic glycoprotein with anti-inflammatory properties, but they do not affect monocyte chemotaxis. $^{7}$ The inhibition of neutrophil recruitment by apoptotic cells represents an effective mechanism for limiting tissue injury and inflammation. It is known that the recruited neutrophils and the release of their proteolytic enzymes contribute to many pathological inflammatory conditions, such as ischemic injury of the heart, ${ }^{8}$ lung $^{9}$ and skeletal muscle, ${ }^{10}$ as well as to toxic insults to the liver ${ }^{11}$ and lung. ${ }^{12}$

It was recently shown that chemotherapeutic agents, such as anthracyclines, can induce an immunogenic form of apoptotic cell death, ${ }^{13,14}$ which stimulates the induction of an adaptive immune response that eradicates tumors. The immunostimulatory properties of these immunogenic apoptotic cells are explained by the action of damage-associated molecular patterns (DAMPs) or cell death-associated molecules

\footnotetext{
${ }^{1}$ Department for Molecular Biomedical Research, VIB, Ghent, Belgium; ${ }^{2}$ Department of Biomedical Molecular Biology, Ghent University, Ghent, Belgium; ${ }^{3}$ Upper Airway Research Laboratory, Department of Oto-Rhino-Laryngology, Ghent University Hospital, Ghent, Belgium; ${ }^{4}$ Laboratory for Cellular and Molecular Immunology, Vrije Universiteit Brussel, Brussels, Belgium and ${ }^{5}$ Department of Molecular and Cellular Interactions, VIB, Brussels, Belgium

${ }^{*}$ Corresponding author: P Vandenabeele, Department for Molecular Biomedical Research, VIB-Ghent University, Technologiepark 927, B-9052 Ghent (Zwijnaarde), Belgium. Tel: + 329 3313760; Fax: + 329 3313609; E-mail: Peter.Vandenabeele @dmbr.UGent.be

${ }^{6}$ These authors share first authorship.

Keywords: doxorubicin; TLRs; apoptosis; neutrophils; DAMPs; immunogenic cell death

Abbreviations: AnnV, annexin V; ATP, adenosine triphosphate; CDAM, cell death-associated molecule; CRT, calreticulin; DAMP, damage-associated molecular pattern; HMGB1, high mobility group box 1 protein; HSP, heat shock protein; i.p., intraperitoneal; IL, interleukin; LPS, lipopolysaccharide; MCP-1, monocyte chemotactic protein-1; MSU, monosodium urate; MTC, mitomycin c; MYD88, myeloid differentiation primary response gene 88; NLR, the nucleotide-binding oligomerization domain (NOD)-like receptor; PAMP, pathogen-associated molecular pattern; PEC, peritoneal exudate cell; PRR, pattern-recognition receptor; RLR, RIG-I-like receptor; TGF- $\beta$, transforming growth factor beta; TLR, Toll-like receptor; TRIF, Toll/LL-1R domain-containing adaptor inducing IFN- $\alpha$; UTP, uridine triphosphate

Received 07.7.10; revised 22.12.10; accepted 04.1.11; Edited by P Mehlen; published online 11.2.11
} 
(CDAMs). ${ }^{15,16}$ DAMPs are mostly intracellular molecules that acquire immunostimulatory activity following their secretion or release by stressed or dying cells. Thus, immunogenic apoptotic cell death is characterized by surface exposure of calreticulin (CRT) ${ }^{17}$ and release of high mobility group box 1 protein (HMGB1) from the nucleus of dying tumor cells into the extracellular space. ${ }^{13}$ Interestingly, DAMPs are recognized by host cells expressing a set of receptors known as pattern-recognition receptors (PRRs), such as Toll-like receptors (TLRs), the nucleotide-binding oligomerization domain (NOD)-like receptors (NLRs) and RIG-I-like receptors (RLRs). ${ }^{18}$ When these receptors are triggered by cells dying in an immunogenic or non-physiological way, they rapidly initiate host defense responses. For example, it has been shown that HMGB1 is required for the induction of a doxorubicin-induced anti-tumor immune response in a TLR-4/myeloid differentiation primary response gene 88 (MyD88)-dependent pathway in mice. ${ }^{13}$ Several reports address the interaction of the adaptive immune system with immunogenic apoptotic cells, but it is not fully understood how the cells of the innate immune system, such as neutrophils, react to immunogenic apoptotic cells.

Given that doxorubicin induces immunogenic apoptotic cell death and that classical apoptotic cells preferentially stimulate monocyte recruitment, we sought to determine how cells killed by doxorubicin are sensed by the innate immune system. We established an in situ murine model of apoptosis induction by intraperitoneal (i.p.) injection of doxorubicin. I.p. injection of doxorubicin resulted in the generation of mostly apoptotic monocytes/macrophages and induced an acute inflammatory response in the peritoneal cavity characterized by recruitment of neutrophils and production of IL- 6 and monocyte chemotactic protein-1 (MCP-1). This acute inflammatory response was specific to immunogenic chemotherapeutics because a non-immunogenic drug, mitomycin C (MTC), also induced cell death in the peritoneal cavity but was incapable of eliciting neutrophil attraction. We found that MyD88 is essential for the doxorubicin-induced acute inflammatory response and that it is required primarily as an adaptor molecule in the TLR-2 and TLR-9 signaling pathways.

\section{Results}

Acute inflammation induced by doxorubicin is associated with apoptosis of monocytes/macrophages. I.p. injection of doxorubicin resulted in an acute inflammatory response accompanied by the influx of neutrophils and an increase in the levels of IL-6 and MCP-1 in the lavage fluid collected $16 \mathrm{~h}$ after doxorubicin injection (Figure 1). The number of annexin $\mathrm{V}$ (AnnV)-positive and Sytox-negative a

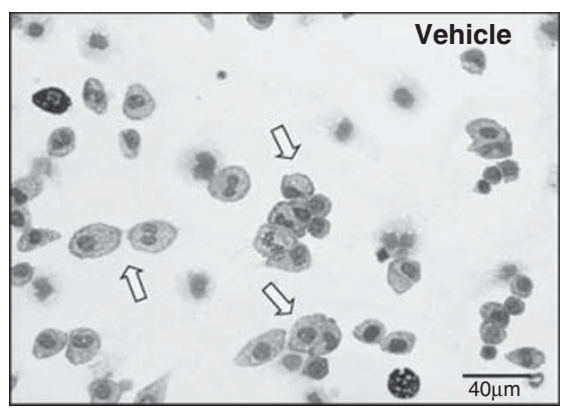

C

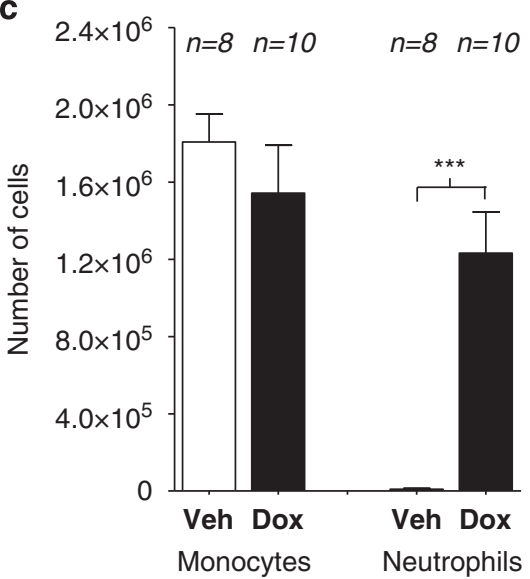

b
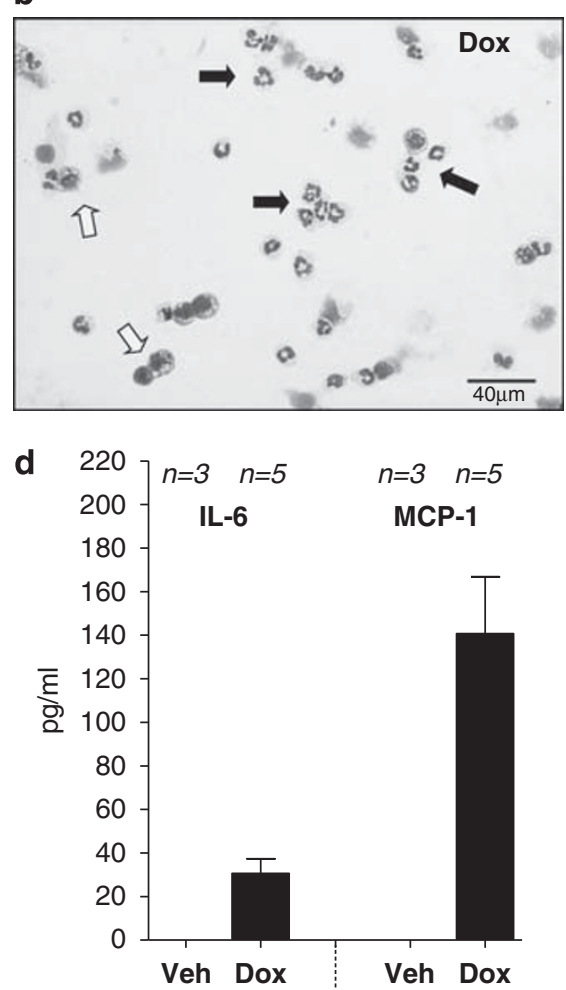

Figure 1 I.p. injection of doxorubicin (10 mg/kg) induces a sterile inflammatory response. (a and $\mathbf{b})$ Representative image of May-Grünwald and Giemsa staining of PECs from C57BL/6 wild-type mice $16 \mathrm{~h}$ after i.p. injection of doxorubicin. White and black arrows point to monocytes/macrophages and neutrophils, respectively. Bars, $40 \mu \mathrm{m}$. (c) Some neutrophils among PECs of wild-type mice injected with doxorubicin or vehicle. (d) Concentration of IL-6 and MCP-1 in the peritoneal lavage fluids of wild-type mice injected with $10 \mathrm{mg} / \mathrm{kg}$ doxorubicin or vehicle (control). Means and S.E.M. are for pooled data, and $n$ is the total number of mice in each group; ${ }^{* \star \star} P<0.0001$ 

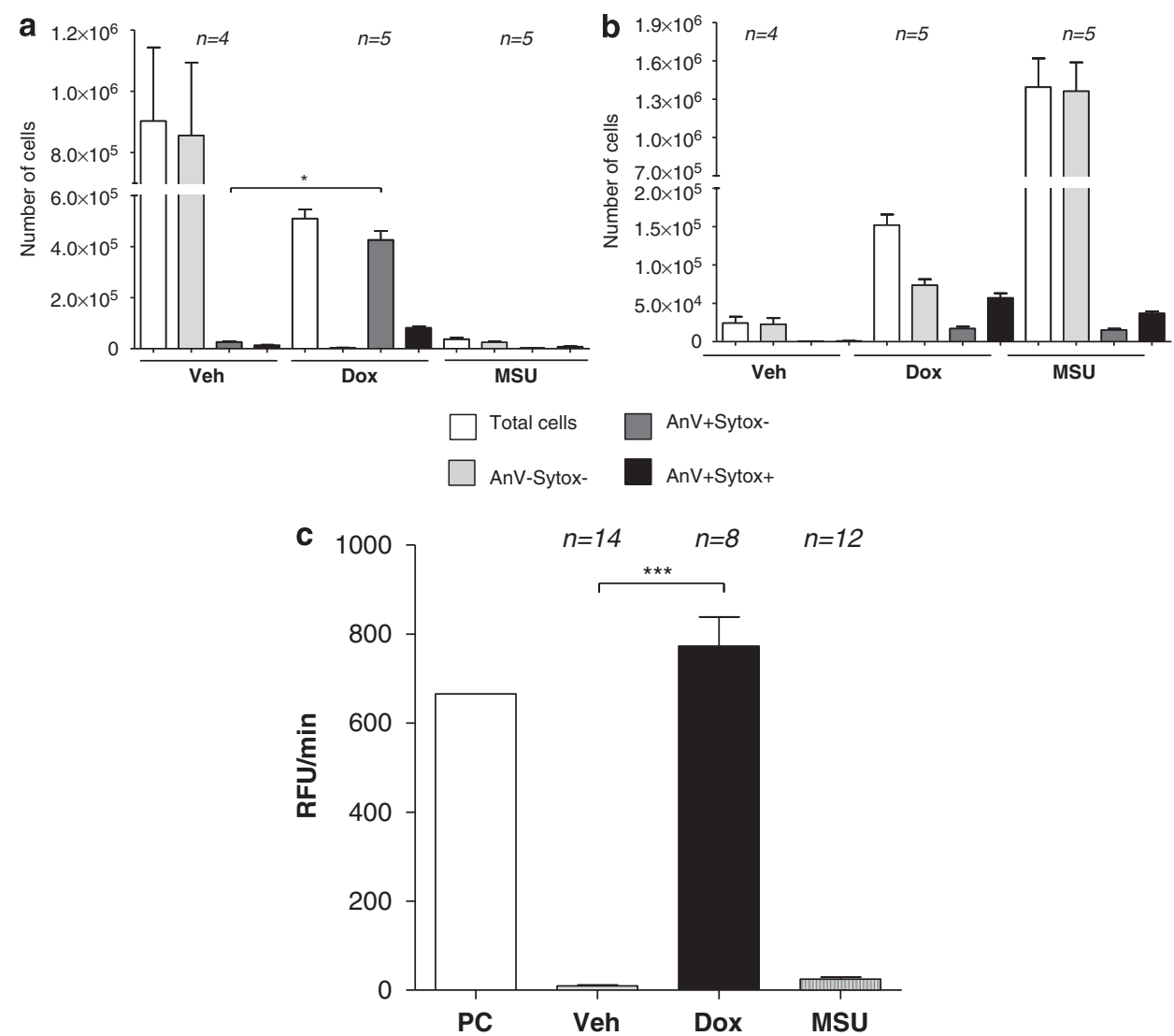

Figure 2 I.p. injection of doxorubicin induces apoptosis of monocytes/macrophages. (a) Identification of macrophages/monocytes $\left(\mathrm{F} 4 / 80^{+} \mathrm{CD} 11 \mathrm{~b}^{+}\right.$, total cells) and (b) neutrophils $\left(\mathrm{CD} 11 \mathrm{~b}^{+} \mathrm{Ly}-6 \mathrm{G}^{+}\right.$, total cells) in combination with phosphatidylserine staining and detection of plasma membrane permeabilization by AnnV and Sytox, respectively. Cells that were $\mathrm{AnnV}^{-}$Sytox ${ }^{-}, \mathrm{AnnV}^{+}$Sytox ${ }^{-}, \mathrm{AnnV}^{+}$Sytox ${ }^{+}$were indentified; these cells represent viable, early apoptotic and secondary necrotic cells, respectively. The experiment was repeated two times, and the representative data are shown. (c) Increased DEVDase activity in PECs $6 \mathrm{~h}$ after doxorubicin injection. Of note, the same PECs were used for measurement of DEVDase activity and for phenotyping by flow cytometry. PECs were isolated $6 \mathrm{~h}$ after i.p. injection of either $10 \mathrm{mg} / \mathrm{kg}$ of doxorubicin or $2 \mathrm{mg} / \mathrm{mouse}$ of MSU. These data represent the values of two pooled experiments. Means and S.E.M. are for pooled data, and $n$ is the total number of mice in each group; PC is a positive control for the activity of the recombinant caspase-3 (150 ng). RFU/min, relative fluorescence units per minute. ${ }^{*} P<0.01$; ${ }^{* * *} P<0.0001$. Negative controls were injected with vehicle alone

$\left(\mathrm{AnnV}^{+}\right.$Sytox $\left.^{-}\right)$cells increased in the peritoneum $6 \mathrm{~h}$ after i.p. injection of doxorubicin (Figure 2a), indicating that the majority of peritoneal exudate cells (PECs) were in early stages of apoptosis. To further confirm the type of cell death, caspase activity was determined in the PECs. We found that DEVDase activity (caspase-3/7) was increased in these PECs at $6 \mathrm{~h}$ (Figure 2c), confirming that they were dying by apoptosis. By performing multi-color flow cytometry, we found that the majority of cells that died apoptotically due to doxorubicin treatment were mainly monocytes/macrophages with some minor neutrophils (Figures 2a and b). Moreover, to exclude the possibility that the observed apoptotic cells were not just regular dying neutrophils, we injected i.p. monosodium urate (MSU), which induces strong neutrophil recruitment. $^{22} \mathrm{MSU}$ induced significantly more neutrophil attraction than doxorubicin, but again the number of apoptotic neutrophils in the peritoneal cavity was negligible. Also, no caspase3/7 activity was measured in PECs after i.p. injection of MSU (Figure 2c). All these data indicate that monocytes/macrophages represent the major cell population that dies by apoptosis after i.p. injection of doxorubicin.
To determine whether neutrophil attraction is induced by the presence of apoptotic cells in the peritoneum, we performed a dose titration experiment in which we investigated the relation between the percent of Sytox ${ }^{+}$cells and the number of attracted neutrophils as a function of doxorubicin dose. We observed a dose-dependent increase in the percentage of Sytox $^{+}$cells (Figures $3 a$ and $c$ ). Moreover, there was a linear correlation between the percent of Sytox ${ }^{+}$cells and neutrophil influx (Figure 3b). Notably, the recruitment of neutrophils increased slowly; it was high at $16 \mathrm{~h}$ and reached a maximum at $38 \mathrm{~h}$, which is considerably later than the peak of cell death observed at $6 \mathrm{~h}$ (Figure $3 \mathrm{~d}$ ). This time lag between the peak of dead cells and the maximum number of neutrophils implies that neutrophils are attracted after the induction of cell death and that this recruitment is associated with cell death. We used this model to investigate how doxorubicin stimulates acute inflammation in the peritoneum.

Acute inflammation induced by doxorubicin is specific for immunogenic chemotherapeutics. To determine whether an acute inflammatory response, as judged by 
a
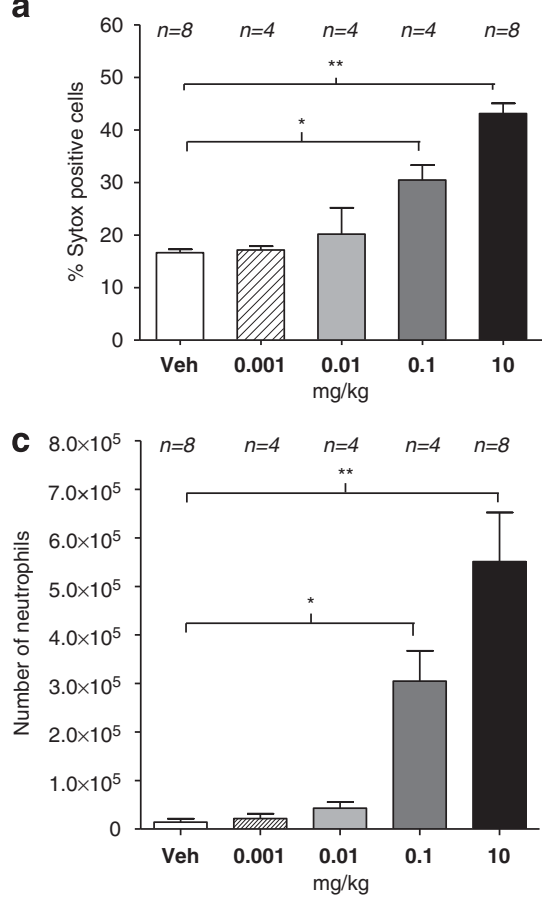

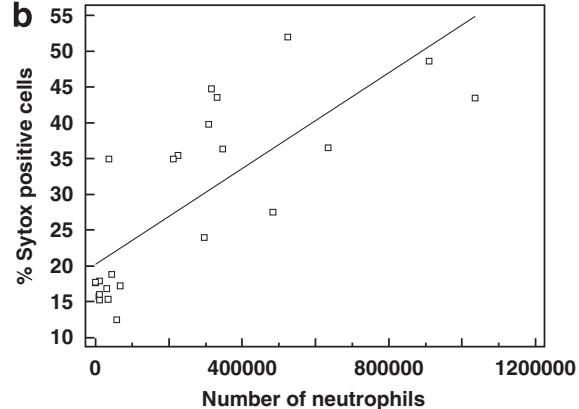

d
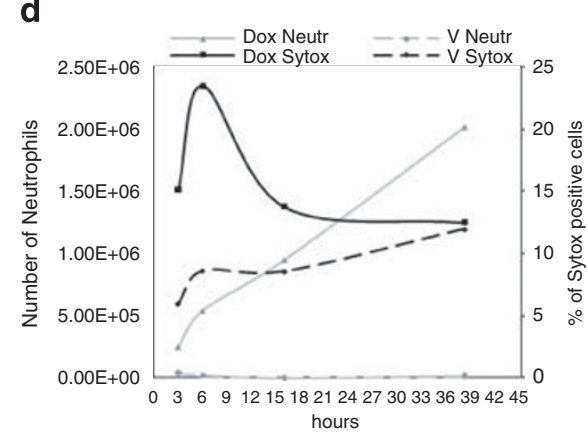

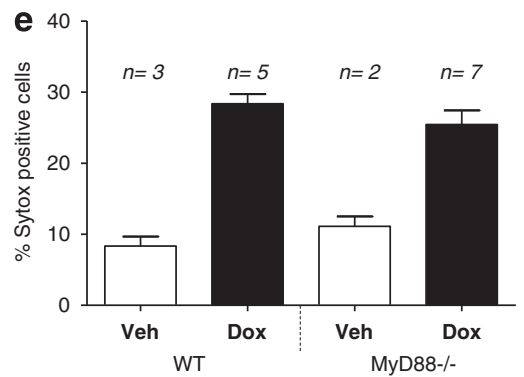

Figure 3 Sterile inflammatory response induced by doxorubicin is associated with apoptosis. (a) Dead cells were identified by Sytox staining of the recovered PECs $6 \mathrm{~h}$ after injection of the indicated doses of doxorubicin (per kg). (b) Linear correlation of neutrophil counts and percent of Sytox ${ }^{+}$cells. $(R=0.770 ; P<0.001)$. (c) The number of neutrophils recruited $6 \mathrm{~h}$ after injection of the indicated doses of doxorubicin (per $\mathrm{kg}$ of body weight). (d) Kinetics of cell death and neutrophil (neutr) recruitment at 3,6,16 and $38 \mathrm{~h}$. The number of Sytox ${ }^{+}$cells was maximal $6 \mathrm{~h}$ after doxorubicin (dox) injection, and then it declined and returned gradually to that seen in control mice (injected with vehicle (V)). However, recruitment of neutrophils gradually increased and reached the maximum at $38 \mathrm{~h}$. There is a clear time lag between the maximum number of dead cells and the maximum number of attracted neutrophils. (e) Percent of Sytox ${ }^{+}$-positive cells in PECs $6 \mathrm{~h}$ after i.p. injection of doxorubicin (10 mg/kg) in wild-type and MyD88 ${ }^{-1-}$ mice. Means and S.E.M. are for pooled data, and $n$ is the total number of mice in each group. ${ }^{*} P<0.02 ;{ }^{\star} P<0.004$. Negative controls were injected with vehicle alone

neutrophil attraction, is specific to the immunogenic anthracyclines, we injected i.p. MTC, which induces a non-immunogenic cell death. ${ }^{17}$ Six hours after i.p. injection of MTC, there was a significant increase in Sytox ${ }^{+}$cells (Figure 4a), but no increase in neutrophil attraction was detected (Figure $4 b$ ). This result indicates that the acute inflammatory response induced by doxorubicin is specific for immunogenic chemotherapeutics.

The role of TLRs in doxorubicin-induced acute inflammation. TLRs recognize signature molecules derived either from pathogens (pathogen-associated molecular patterns, PAMPs) or from dead or damaged cells (DAMPs or CDAMs). When TLRs recognize their ligands, they signal through the TIR adaptor protein MyD88, except for TLR-3, which uses the Toll/IL-1R domain-containing adaptor inducing IFN- $\alpha$ (TRIF). To determine whether TLRs are involved in the inflammation triggered by doxorubicin, we injected doxorubicin i.p. in mice deficient in MyD88 (encoded by MyD88) and mice mutant for TRIF ${ }^{\text {Lps2 }},{ }^{19}$ and the acute inflammatory response was evaluated by quantifying the influx of neutrophils. After $16 \mathrm{~h}$, wild-type and TRIFLps2 mice had abundant neutrophils in their abdominal cavities, but this

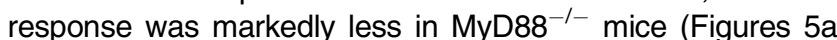
and b). Remarkably, the acute inflammatory response in MyD88-deficient mice was reduced by 22 -folds at $16 \mathrm{~h}$ (Figure 5a). Consistent with the reduced neutrophil infiltration in MyD88-deficient mice, the production of IL-6 and MCP-1 was reduced by $12-$ and 6.5-folds, respectively (Figure 6b). The percentage of Sytox ${ }^{+}$cells in the MyD88-deficient mice was not different from that in wild-type mice (Figure $3 e$ ), indicating that the cell death process itself was not affected.

The requirement for MyD88 in doxorubicin-induced inflammation suggested that TLRs might be involved. Therefore, 

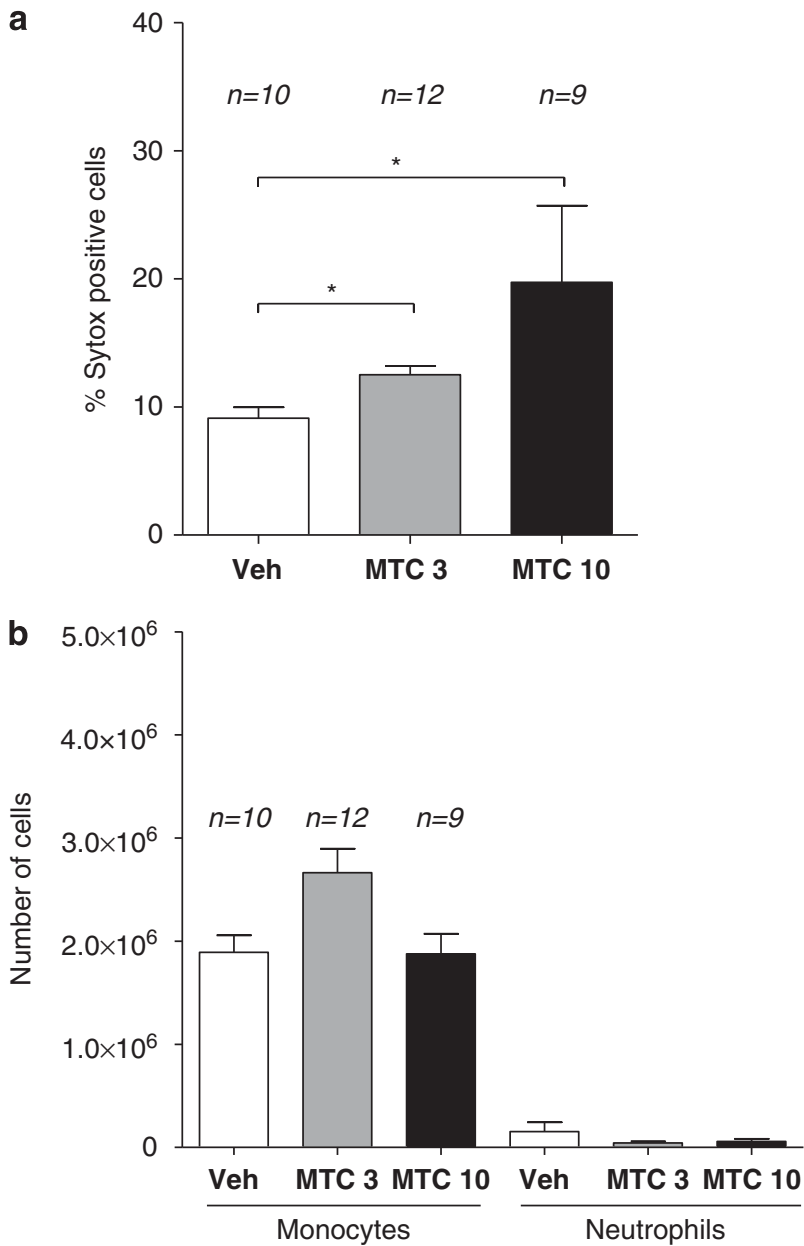

Figure 4 I.p. injection of MTC does not induce neutrophil recruitment. (a) Dead cells were identified by Sytox staining of the recovered PECs $6 \mathrm{~h}$ after injection of MTC $3 \mathrm{mg} / \mathrm{kg}$ (MTC 3) or $10 \mathrm{mg} / \mathrm{kg}$ (MTC 10). (b) The number of monocytes and neutrophils recruited $6 \mathrm{~h}$ after i.p. injection of MTC at $3 \mathrm{mg} / \mathrm{kg}$ or $10 \mathrm{mg} / \mathrm{kg}$. Means and S.E.M. are for pooled data, and $n$ is the total number of mice in each group. ${ }^{*} P<0.02$. Negative controls were injected with vehicle alone

we injected doxorubicin into mice deficient in various TLRs (TLR-2, TLR-3, TLR-4 or TLR-9). Statistical analysis of the results showed no significant reduction in neutrophil infiltration in mice deficient in TLR-3 or TLR-4 compared with wild-type animals (Figures $5 \mathrm{~d}$ and $\mathrm{e}$ ). However, the neutrophil response to doxorubicin-induced acute inflammation in TLR-2 and TLR-9 mutant mice was reduced about twofold (Figures $5 \mathrm{c}$ and $\mathrm{f}$ ), implying that these TLRs fulfill an essential role in the recruitment of neutrophils following an apoptotic insult by doxorubicin. However, we do not know whether TLR-1, TLR-5, TLR-6, TLR-7 and TLR-11 (for which we had no knockout mice available) are also involved.

The role of NLRs in doxorubicin-induced acute inflammation. Recently, another family of sensors of PAMPs and DAMPs was identified; this family includes NLRs. ${ }^{23}$ However, although TLRs mediate recognition at the cell surface and at endosomes, NLRs induce innate immune responses on recognition of PAMPs and DAMPs in the cytosol. Several NLRs form a caspase-1-activating multiprotein complex, termed the inflammasome, which processes pro-inflammatory cytokines, including IL-1 $\beta$. It has been shown that dying tumor cells release ATP, which then acts on $\mathrm{P}_{2} \mathrm{X}_{7}$ purinergic receptors from DCs and triggers the NOD-like receptor family, pyrin domain containing3 protein (NLRP3)-dependent caspase-1 activation complex; this allows secretion of IL-1 $\beta .^{24}$ To investigate whether the inflammasome is important in the doxorubicin-induced neutrophil influx, we injected doxorubicin in caspase-1deficient mice. The acute inflammatory response in caspase-1-deficient mice was not significantly weaker than in wild-type animals (Figure 6a). Therefore, inflammasomes are probably not involved in the induction of the acute inflammatory response to doxorubicin.

TLR-9 antagonist reduces doxorubicin-induced acute inflammation. Having demonstrated that attraction of neutrophils is reduced in TLR- $9^{-1-}$ mice, we tested whether acute inflammation can be reduced in wild-type mice by the administration of a TLR-9 antagonist. Wild-type mice were injected i.p. with the active TLR-9 antagonist ODN2088 $(50 \mu \mathrm{g})$ or the inactive control $(50 \mu \mathrm{g})$ together with doxorubicin, and $6 \mathrm{~h}$ later with inactive or active ODN. Recruited cells were phenotyped $16 \mathrm{~h}$ after doxorubicin injection. The active TLR-9 antagonist significantly reduced the recruitment of neutrophils but the inactive control did not (Figure 6c). This further confirms the importance of TLR-9 in doxorubicin-induced acute inflammation and also identifies a new therapeutic strategy that might be used to limit the inflammatory side effects of treating peritoneal carcinomatosis by i.p. injection of doxorubicin.

HMGB-1 does not contribute to doxorubicin-induced acute inflammation. As HMGB1 is one of the DAMPs that could act via TLR-2 $2^{25,26}$ and TLR-9, ${ }^{27,28}$ which are implied in doxorubicin-induced acute inflammation in our model, we measured the concentration of HMGB1 in the peritoneal lavage. We found that the concentration of HMGB1 in the peritoneal lavage 3, 6 and $16 \mathrm{~h}$ after doxorubicin injection was not significantly different from that after injection of vehicle (Figure 6d).

\section{Discussion}

Identifying the signaling pathways involved in the sensing of dead or stressed cells that contribute to the immunomodulatory nature of cell death is critical to our understanding of this fundamental biological process. Apoptosis and necrosis are two major forms of cell death. ${ }^{29}$ Classically, apoptosis was described as an immunologically silent and anti-inflammatory form of cell death distinct from necrosis,,$^{1,2}$ whereas necrosis was described as causing stimulation of the immune system. ${ }^{30}$

We established a model for inducing apoptotic cell death in situ by i.p. injection of an anthracycline chemotherapeutic, namely doxorubicin. We show that injection of doxorubicin into the peritoneum induces a sterile inflammatory response characterized by the induction of apoptosis, recruitment of 
a

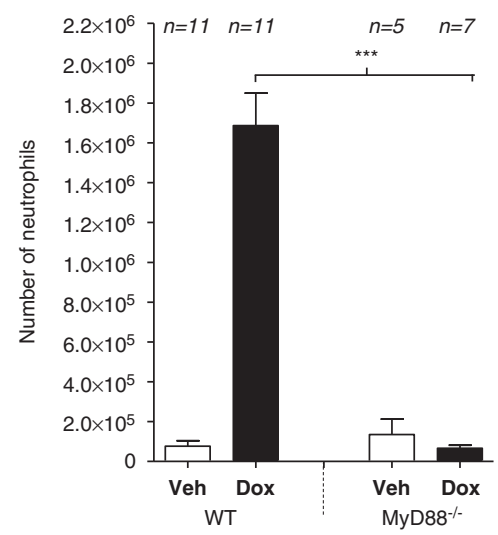

c
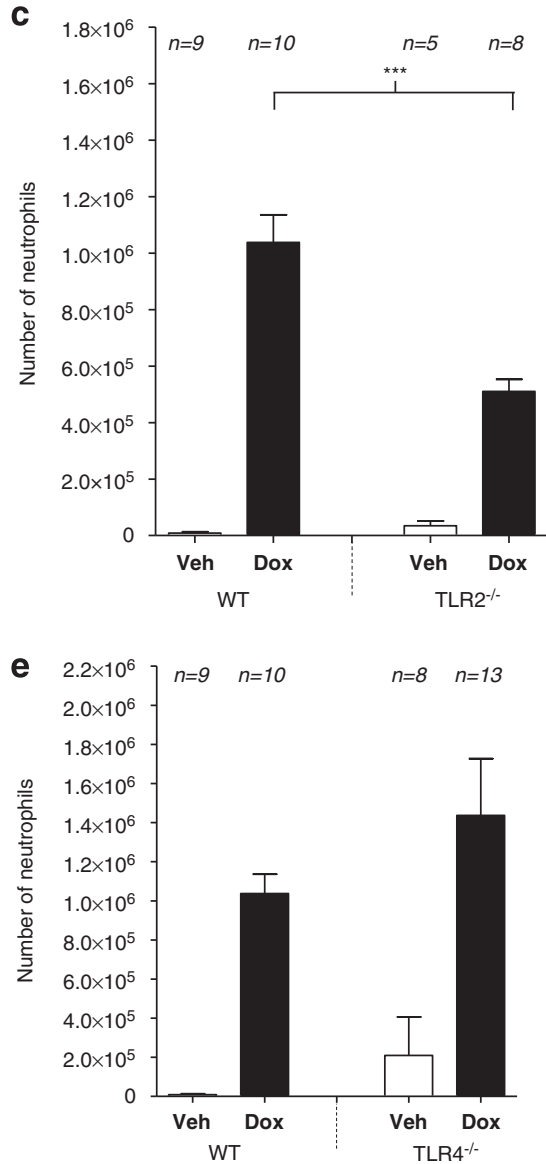
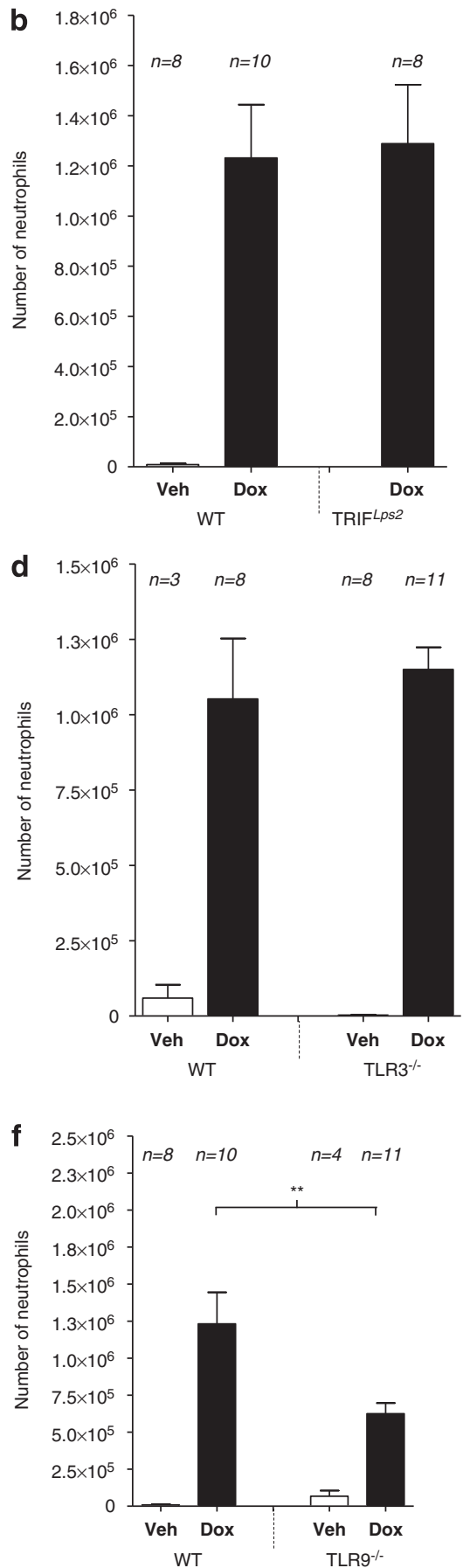

Figure 5 MyD88, TLR-2 and TLR-9 are required for doxorubicin-induced neutrophil recruitment, but TRIF, TLR-3 and TLR-4 are not. Numbers of neutrophils in PECs

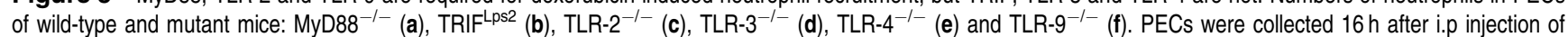
doxorubicin at $10 \mathrm{mg} / \mathrm{kg}$. Means and S.E.M. are for pooled data, and $n$ is the total number of mice in each group; ${ }^{\star \star \star} P<0.0001 ;{ }^{* \star} P<0.004$. Negative controls were injected with vehicle alone

neutrophils and production of pro-inflammatory mediators, such as IL-6 and MCP-1. Monocytes/macrophages represent a major target of doxorubicin. Six hours after i.p. injection of doxorubicin, most of the monocytes/macrophages were in early apoptosis (AnV ${ }^{+}$Sytox). Increased DEVDase activity in the PECs pointed to enhanced activity of caspase- 3 and -7 , which are crucial executioners of apoptosis. ${ }^{21}$ This agrees with a previous report on doxorubicin-induced apoptosis accompanied by activation of caspase- 3 in the human ovarian cancer cell line $\mathrm{A} 2780^{31}$ and in the murine colon carcinoma CT26 line. ${ }^{14}$ The kinetic experiments showed that there was a time lag between the appearance of the Sytox ${ }^{+}$cells (at $6 \mathrm{~h}$ ) and neutrophil influx (at 16-38h). Moreover, influx of neutrophils was dose dependent and linearly correlated 

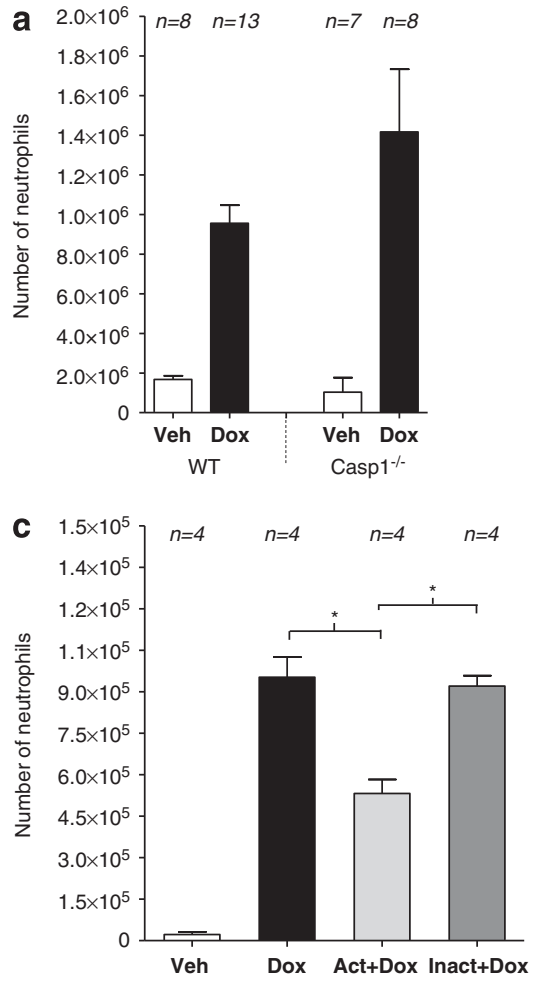

b

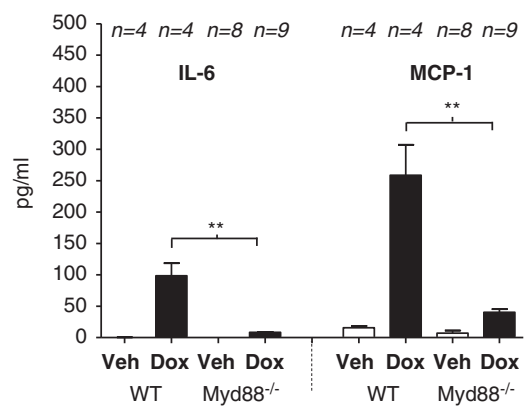

d

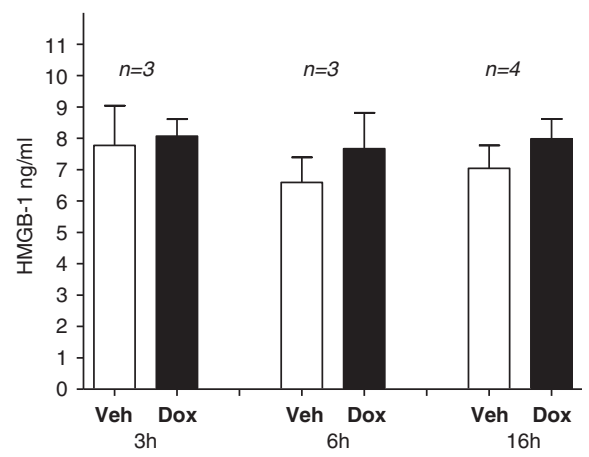

Figure 6 Role of caspase-1 in neutrophil recruitment and the effect of a TLR-9 antagonist. (a) Caspase-1 is not required for the sterile inflammatory response triggered by doxorubicin. Numbers of neutrophils in PECs of wild-type and caspase-1 ${ }^{-1-}$ mice. PECs were collected $16 \mathrm{~h}$ after i.p injection of doxorubicin. (b) Concentration of IL-6 and MCP-1 in the peritoneal lavage fluids of wild-type and MyD88 $8^{-1-}$ mice injected with doxorubicin or vehicle. (c) Treatment of wild-type mice with a TLR-9 antagonist (ODN2088) significantly reduced the recruitment of neutrophils $16 \mathrm{~h}$ after i.p. injection of doxorubicin. Inactive antagonist used as a control had no effect on neutrophil recruitment. (d) Concentration of HMGB-1 was measured in the peritoneal lavage fluids of wild-type mice 3, 6 and $16 \mathrm{~h}$ after injection of vehicle or doxorubicin. Doxorubicin was injected at $10 \mathrm{mg} / \mathrm{kg}$. Means and S.E.M. are for pooled data, and $n$ is the total number of mice in each group; ${ }^{\star} P<0.02$; ${ }^{\star \star} P<0.004$. Negative controls were injected with vehicle alone

( $r=0.770 ; P<0.001)$ with the percentage of Sytox ${ }^{+}$cells. All these data suggest that the recruitment of neutrophils is associated with apoptotic cells that are killed in situ by doxorubicin. Of note, it has been recently shown that doxorubicin does not have a direct immunostimulatory activity on antigen-presenting cells, ${ }^{32}$ thereby excluding a direct effect of doxorubicin on the host cells.

Only some apoptotic stimuli, including anthracyclines, induce an immunogenic form of apoptosis characterized by surface exposure of CRT and release of HMGB1. ${ }^{17}$ When immunogenic apoptotic cancer cells are implanted into immunocompetent mice, they can convert the 'classical' silent nature of apoptosis into an 'immunogenically active' form of apoptosis. To determine whether only immunogenic chemotherapeutics induce the acute inflammatory response induced by doxorubicin, we injected i.p. MTC, which induces a non-immunogenic form of apoptosis. Indeed, we found that this treatment caused substantial cell death but did not induce neutrophil attraction. These data indicate that the acute inflammatory response induced by doxorubicin is specific for immunogenic chemotherapeutics.

'Classical' apoptotic cells stimulated with traditional apoptotic stimuli, such as anti-Fas antibodies or UV, are sensed by monocytes, which are then recruited in a $\mathrm{P}_{2} \mathrm{Y}_{2}$-receptordependent manner because of the release of ATP and UTP. ${ }^{6}$
Apoptotic cells secrete signals, such as lactoferrin, that inhibit the migration of neutrophils but not of monocytes. ${ }^{7}$ In contrast, we show that apoptotic cells killed by doxorubicin induce an acute inflammatory response characterized by recruitment of neutrophils but have no significant effect on recruitment of monocytes. Thus, apoptotic cells challenged with doxorubicin behave like necrotic cells in terms of neutrophil recruitment. Indeed, it has been shown in a model of sterile inflammation that i.p. injection of heat-killed necrotic cells induces a strong acute inflammation characterized by recruitment of neutrophils. However, in contrast to our results, Chen et al. ${ }^{33}$ showed that the IL-1R-MyD88 pathway is important for neutrophil attraction while TLRs are not. The release of IL-1 $\alpha$ from necrotic cells was required for production of CXCL- 1 and IL-6 by mesothelial cells, which was followed by neutrophil recruitment. ${ }^{34}$ In another study, using a model of bacterial peritonitis induced by cecal ligation and puncture, RNA from necrotic cells could amplify the inflammatory response in a TLR-3-dependent manner. ${ }^{35}$ However, in our study, recruitment of neutrophils was independent of TLR-3, and in contrast to the Cavassani study, it required the functions of TLR-2 and TLR-9. The discrepancies between our results and the work of Chen et al. ${ }^{33}$ and Cavassani et al. ${ }^{35}$ could be explained by differences in the form of cell death (apoptosis versus primary necrosis) and death stimuli (doxorubicin versus heat shock or 
ischemia in combination with bacterial peritonitis). The form of cell death and the nature of the stimulus might determine which pathway is used in sensing dead cells.

Some NLRs are involved in the recognition of DAMPs released from dead or stressed cells. This recognition can lead to activation of caspase- 1 through the assembly of a cytosolic protein complex known as the 'inflammasome'. It has been shown that dying tumor cells release ATP, which then acts on $\mathrm{P}_{2} \mathrm{X}_{7}$ purinergic receptors from $\mathrm{DCs}$ and triggers the NLRP3-dependent caspase-1 activation complex. ${ }^{24}$ Therefore, we became interested in the possible involvement of caspase-1 in the doxorubicin-induced acute inflammatory response in the peritoneum. However, unexpectedly, the acute inflammatory response in caspase-1-deficient mice was comparable to that in wild-type mice. Similarly, the level of $\mathrm{IL}-1 \beta$ in the peritoneum was not increased after doxorubicin injection in wild-type mice (data not shown). These results indicate that it is unlikely that inflammasomes are involved in the doxorubicin-induced acute inflammatory response.

The presence of both apoptotic and secondary necrotic cells in our model means that release of some DAMPs could contribute to the sterile inflammatory response induced by doxorubicin. As TLR-3 was not involved in neutrophil recruitment in our model, it is unlikely that RNA acts as a DAMP. But heat shock proteins (HSPs), a family of highly conserved chaperone proteins, do act as DAMPs when exposed on the surface of dead or stressed cells and when they are released. ${ }^{36} \mathrm{HSPs}$ are strong immunostimulants and induce neutrophil recruitment. However, several studies indicate that HSPs are recognized mainly by TLR-4. ${ }^{37,38}$ Activation of immune cells by HSPs (and possibly by other DAMPs) via TLR-4 may raise concerns about endotoxin contamination masking the observed immunostimulatory effects. In our model, neutrophil attraction induced by apoptotic cells was independent of TLR-4, which excludes the involvement of HSP60, HSP70 and HSP72. The absence of TLR-4 involvement also excludes the possibility that contaminating lipopolysaccharide (LPS)-like endotoxins contributed to the doxorubicin-induced inflammation.

HMGB1 is another potential DAMP that could be released from necrotic ${ }^{39}$ and secondary necrotic cells. ${ }^{13}$ HMGB1 acts on several receptors, including TLR- ${ }^{25}$ and TLR-9. ${ }^{27}$ Both TLR-2 and TLR-9 are expressed in various cell types, including neutrophils, monocytes, T cells, B cells, NK cells and dendritic cells. ${ }^{40}$ In our model, the concentration of HMGB1 in the peritoneal lavage 3, 6 and $16 \mathrm{~h}$ after doxorubicin injection was not significantly different from that after injection of vehicle. Probably, most apoptotic cells are engulfed before going into secondary necrosis, which may exclude the possible release of HMGB1 from secondary apoptotic cells in our model system. In contrast, previous work has shown that HMGB1 is released from secondary necrotic (late apoptotic) tumor cells in response to doxorubicin treatment. ${ }^{13}$ This release was implicated in the establishment of an anti-tumor adaptive immune response in a TLR-4dependent manner. Here, we show that apoptotic/secondary necrotic cells killed in situ by doxorubicin stimulate neutrophil influx independently of TLR-4.

We also show that the sterile neutrophil inflammation induced by doxorubicin can be blocked by a specific TLR-9 antagonist. Further studies are needed to investigate whether blockade of the TLR-2 and TLR-9 pathways could prevent the sterile inflammation induced by doxorubicin without markedly affecting its anti-proliferative effects.

Together, our findings provide important new insights into how the innate immune system senses apoptotic cells killed by immunogenic chemotherapeutic agents. We show that the acute inflammatory response induced by doxorubicin is specific to immunogenic chemotherapeutics and is associated with apoptosis of monocytes/macrophages. Apoptotic cells killed in situ by doxorubicin are a potential source of DAMPs, which can stimulate TLR-2 and TLR-9 and induce acute inflammation. Our data clearly demonstrate that the TLR-2/TLR-9-MyD88 signaling pathways have a central role in initiating the acute inflammatory response to apoptotic cells induced by doxorubicin administration. We could not identify the DAMPs involved in this process, and it is possible that new molecules are involved in the induction of the acute inflammatory process induced by apoptotic/secondary necrotic cells killed in situ.

\section{Materials and Methods}

Mice. For the kinetics and dose-response experiments, female C57BL/6 and Balb/c mice (8-10 weeks old) were purchased from Janvier (Bio Services BV, Uden, The Netherlands). The following mutant mice were used: $M y d 88^{-1-}$ (Balb/c background); $T L R-2^{-1-}$, TLR- $4^{-1-}$ and TLR- $9^{-1-}$ (C57BL/6 background); TLR-3 ${ }^{-1-}$ (B6129 background); and caspase- $1^{-1-}$ (6x back crossed to C57Bl/6). Mutant $T R I F^{L p s 2}$ mice (C57BL/6 background) were generated by random mutagenesis with ENU; these mice have a distal frameshift error in a TRIF protein, which impairs its function. ${ }^{19}$ For experiments with knockout mice, wild-type mice of appropriate background were used, and they were bred under the same animal house conditions as the others. Mice were housed in a SPF facility with $12-12 \mathrm{~h}$ light-dark cycles and received water and food ad libitum. All experimental procedures were approved by the local Ethics Committee of Ghent University-VIB. Although some mutants were of Balb/c and mixed B6129 genetic backgrounds, we found that doxorubicin-induced sterile inflammatory responses were not different in C57BL/6, Balb/c and B6129 mice (data not shown).

Doxorubicin-induced inflammation in vivo. Doxorubicin hydrochloride (Sigma-Aldrich, Bornem, Belgium) was freshly dissolved in sterile LPS-free $0.9 \%$ $\mathrm{NaCl}$ (Braun-NVSA, Diegem, Belgium) at $5 \mathrm{mM}$. Aliquots were frozen and used before each experiment to ensure constant quality. Mice were injected i.p. with $10 \mathrm{mg} / \mathrm{kg}$ of doxorubicin (or as otherwise indicated) in $0.2 \mathrm{ml}$ of $0.9 \% \mathrm{NaCl}$. Equal volumes of $0.9 \% \mathrm{NaCl}$ were injected as negative controls. At different time points, animals were euthanized by $\mathrm{CO}_{2}$ exposure, and their peritoneal cavities were washed with $8 \mathrm{ml}$ of sterile PBS. The peritoneal lavages were centrifuged at $450 \mathrm{~g}$ for $5 \mathrm{~min}$ and supernatants were stored at $-20^{\circ} \mathrm{C}$ until analyzed for cytokines. Red blood cells were lysed with ACK cell lysis buffer (Lonza Walkersville, Basel, Switzerland) and the number of PECs was counted in a hematocytometer using Trypan blue. The doxorubicin solution used for injection contained $<42 \mathrm{pg} / \mathrm{ml}$ endotoxin as measured by Pyrochrome Chromogenic Endotoxin Test kit (Nodia, Antwerp, Belgium)

To control specificity of doxorubicin-induced acute inflammation, mice were injected i.p. with 3 or $10 \mathrm{mg} / \mathrm{kg}$ of MTC in $0.2 \mathrm{ml}$ of $0.9 \% \mathrm{NaCl}$. Equal volumes of $0.9 \% \mathrm{NaCl}$ were injected as negative controls. Six hours after injection, PECs were isolated and phenotyped.

Phenotyping of PECs by May-Grünwald-Giemsa staining of cytospins. For differential cell counts, cytospin preparations were made and stained according to the May-Grünwald-Giemsa method, and 250 cells were counted per mouse. Eosinophils, neutrophils, monocytes/macrophages and lymphocytes were identified under a light microscope according to standard morphological criteria. The number of monocytes/macrophages and neutrophils in the PECs was determined by multiplying the total cell numbers by the percentage of monocytes/macrophages and neutrophils, respectively. 
Flow cytometric analysis of PECs phenotypes and cell death. PECs $\left(5 \times 10^{5}\right)$ were incubated with rat anti-mouse antibody 2.4G2 (BD Pharmingen, Erembodegem, Belgium) for $30 \mathrm{~min}$ at $4{ }^{\circ} \mathrm{C}$ to block FcyRIIB/III receptors. As doxorubicin has a broad range of auto-fluorescence, we divided each sample and used two different stainings in order to identify monocytes/ macrophages and neutrophils, and we also examined phosphatidylserine exposure and plasma membrane permeability in both types of cells. In order to quantify monocytes/macrophages, the PECs were stained with anti-mouse antibodies F4/80-APC (clone BM8, eBioscience, Vienna, Austria), CD11b-APCCy7 (clone M1/70, BD Pharmingen) and Annexin-V-Alexa Fluor 488 (AnV, Invitrogen, Merelbeke, Belgium). To identify neutrophils, the PECs were stained with anti-mouse antibodies Ly-6G-APC (clone 1A8, BD Pharmingen), CD11b-APCCy7 (clone M1/70, BD Pharmingen) and Ann-V-Alexa Fluor 488 (Invitrogen). All the stainings were done for $30 \mathrm{~min}$ at $4{ }^{\circ} \mathrm{C}$ in annexin-binding buffer (10 mM HEPES$\mathrm{NaOH}, 1 \mathrm{mM} \mathrm{MgCl}, 2.5 \mathrm{mM} \mathrm{CaCl}_{2}, 5 \mathrm{mM} \mathrm{KCl}, 150 \mathrm{mM} \mathrm{NaCl}$, pH 7.4). Just before flow cytometry analysis on BD LSR-II (BD Biosciences, Erembodegem, Belgium), $1.25 \mathrm{nM}$ of Sytox Blue dead cell stain was added (Invitrogen). Data were acquired and analyzed by BD FACSDiva software (BD Biosciences). Monocytes/macrophages and neutrophil numbers in the PECs were determined by multiplying the total cell numbers by the percentage of $\mathrm{CD} 11 \mathrm{~b}^{+} \mathrm{F} 4 / 80^{+}$and $\mathrm{CD} 11 \mathrm{~b}^{+} \mathrm{Ly} 6 \mathrm{G}^{+}$cells, respectively. For all kinetics experiments, the PECs were stained only with $1.25 \mathrm{nM}$ of Sytox Red dead cell stain (Invitrogen) and analyzed on FACSCalibur (BD Biosciences). Data were acquired and analyzed by CellQuest software (BD Biosciences). As Sytox Red and Sytox Blue stain cells with permeabilized plasma membrane in the same way, we use the term 'Sytox' for both stainings.

Caspase-3/7 enzymatic activity by fluorometry. The fluorogenic substrate assay for caspase-3/7 activity was carried out as described previously. ${ }^{20,21}$ Briefly, PECs were washed in cold PBS and lysed in $150 \mu \mathrm{l}$ of caspase lysis buffer ( $1 \%$ NP-40, $10 \mathrm{mM}$ Tris-HCl, pH 7.4, $10 \mathrm{mM} \mathrm{NaCl}, 3 \mathrm{mM} \mathrm{MgCl}_{2}, 1 \mathrm{mM} \mathrm{PMSF}, 0.3 \mathrm{mM}$ aprotinin and $1 \mathrm{mM}$ leupeptin) supplemented with $1 \mathrm{mM}$ oxidized glutathione to block the catalytic cysteine of caspases and to prevent their activation during lysis. Cell debris was removed by centrifugation and DEVDase activity was determined by incubating $30 \mu \mathrm{l}$ ( $25 \mathrm{ng}$ protein) of the soluble fraction with $20 \mu \mathrm{M}$ of Ac-DEVD-amc in $150 \mu \mathrm{l}$ of cell-free system buffer containing $220 \mathrm{mM}$ mannitol, $68 \mathrm{mM}$ sucrose, $2 \mathrm{mM} \mathrm{MgCl}_{2}$, $2 \mathrm{mM} \mathrm{NaCl}, 2.5 \mathrm{mM} \mathrm{KH}_{2} \mathrm{PO}_{4}, 0.5 \mathrm{mM}$ EDTA, $0.5 \mathrm{mM}$ sodium pyruvate, $0.5 \mathrm{mM} \mathrm{L}$ glutamine, $10 \mathrm{mM}$ HEPES-NaOH (pH 7.4) and $10 \mathrm{mM}$ dithiothreitol. The release of fluorescent 7-amino-4-methylcoumarin was measured for $60 \mathrm{~min}$ at 2-min intervals by fluorometry (excitation at $360 \mathrm{~nm}$ and emission at $480 \mathrm{~nm}$ ) (Cytofluor; PerSeptive Biosystems, Cambridge, MA, USA). The maximal rate of increase in fluorescence was calculated $(\Delta F / \mathrm{min})$.

I.p. injection of MSU. Mice were injected i.p. with $2 \mathrm{mg} / \mathrm{mouse}$ of MSU crystals (Enzo Life Sciences, Zandhoven, Belgium) in $200 \mu$ of PBS. Mice injected with equal volumes of PBS served as negative controls. Six hours after injection, PECs were isolated. They were analyzed for caspase-3/7 activity by fluorometry and phenotyped by flow cytometry, as described above.

Cytokine and chemokine analysis. Immunoreactive levels of MCP-1 and IL-6 were measured in peritoneal lavage by using a cytometric bead array mouse soluble protein flex set system (BD Biosciences). The samples were prepared according to the manufacturer's instructions and analyzed on a FACSCalibur (BD Biosciences). Data were acquired by CellQuest software (BD Biosciences) and analyzed by FCAP array software (Soft Flow Hungary Ltd, Pécs, Hungary).

Injection of TLR-9 antagonist. Wild-type mice were injected i.p. with TLR-9 antagonist (ODN2088, $50 \mu \mathrm{g}$ ) or inactive ODN 2088 (50 $\mu \mathrm{g}$, Invivogen, Toulouse, France) together with doxorubicin, and $6 \mathrm{~h}$ later with inactive or active ODN. PECs were obtained $16 \mathrm{~h}$ after doxorubicin injection for cell phenotyping as described previously.

HMGB1 analysis. HMGB1 expression in mouse serum was determined using a specific anti-HMGB1 ELISA (IBL International, GmbH, Hamburg, Germany) according to the manufacturer's protocol. Briefly, $100 \mu \mathrm{l}$ of sample diluent was combined with $10 \mu \mathrm{l}$ of mouse peritoneal lavage fluid in each well and incubated at $37^{\circ} \mathrm{C}$ for $24 \mathrm{~h}$. Wells were washed five times with wash buffer and incubated for $2 \mathrm{~h}$ at $25^{\circ} \mathrm{C}$ with $100 \mu \mathrm{l}$ of POD-conjugate solution. Wells were washed five times with wash buffer and incubated for $30 \mathrm{~min}$ at room temperature with substrate solution. The reaction was stopped by adding $100 \mu$ l of stop solution to each well and the absorbance was read at $450 \mathrm{~nm}$ (the background was subtracted by measuring absorbance at $570 \mathrm{~nm})$.

Statistics. Data from multiple experiments are presented as mean \pm S.E.M. Mann-Whitney test was used to evaluate the differences between the groups (GraphPad Prism-5 Software, La Jolla, CA, USA).

\section{Conflict of interest}

The authors declare no conflict of interest.

Acknowledgements. We thank Professor S Akira (for TLR- ${ }^{-1-}$ mice),

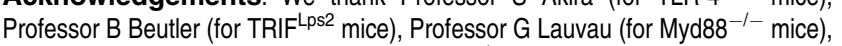
Professor R Beyaert and J Maelfait (for TLR-3 $3^{-1-}$ mice). This work was supported by the Fund for Scientific Research Flanders (FWO-Vlaanderen, 3G072810 to D.V.K.) and by an individual research grant from FWO-Vlaanderen (31507110 to D.V.K). D.V.K is a postdoctoral fellow and AK is a doctoral fellow, both paid by fellowships from FWO-Vlaanderen. Research in the Vandenabeele unit has been supported by Flanders Institute for Biotechnology (VIB), by European grants (FP6 ApopTrain, MRTN-CT-035624; FP7 EC RTD Integrated Project, Apo-Sys, FP7-200767; Euregional PACT II), Belgian grants (Interuniversity Attraction Poles, IAP 6/18) Flemish grants (Fonds Wetenschappelijke Onderzoek Vlaanderen, G.0875.11 and G.0973.11), and Ghent University grants (MRP, GROUP-ID). PV is holder of a Methusalem grant (BOF09/01M00709) from the Flemish Government. We thank Dr. Amin Bredan (DMBR-VIB, Ghent) for editing the paper.

1. Stuart LM, Lucas M, Simpson C, Lamb J, Savill J, Lacy-Hulbert A. Inhibitory effects of apoptotic cell ingestion upon endotoxin-driven myeloid dendritic cell maturation. J Immunol 2002; 168: 1627-1635.

2. Voll RE, Herrmann M, Roth EA, Stach C, Kalden JR, Girkontaite I. Immunosuppressive effects of apoptotic cells. Nature 1997; 390: 350-351.

3. Cvetanovic M, Mitchell JE, Patel V, Avner BS, Su Y, van der Saag PT et al. Specific recognition of apoptotic cells reveals a ubiquitous and unconventional innate immunity. J Biol Chem 2006; 281: 20055-20067.

4. Cvetanovic M, Ucker DS. Innate immune discrimination of apoptotic cells: repression of proinflammatory macrophage transcription is coupled directly to specific recognition. J Immunol 2004; 172: 880-889.

5. Krysko DV, Denecker G, Festjens N, Gabriels S, Parthoens E, D'Herde K et al. Macrophages use different internalization mechanisms to clear apoptotic and necrotic cells. Cell Death Differ 2006; 13: 2011-2022.

6. Elliott MR, Chekeni FB, Trampont PC, Lazarowski ER, Kadl A, Walk SF et al. Nucleotides released by apoptotic cells act as a find-me signal to promote phagocytic clearance. Nature 2009; 461: 282-286.

7. Bournazou I, Pound JD, Duffin R, Bournazos S, Melville LA, Brown SB et al. Apoptotic human cells inhibit migration of granulocytes via release of lactoferrin. J Clin Invest 2009; 119: $20-32$.

8. Romson JL, Hook BG, Kunkel SL, Abrams GD, Schork MA, Lucchesi BR. Reduction of the extent of ischemic myocardial injury by neutrophil depletion in the dog. Circulation 1983; 67: 1016-1023.

9. Hall TS, Breda MA, Baumgartner WA, Borkon AM, Brawn J, Hutchins GM et al. The role of leukocyte depletion in reducing injury to the lung after hypothermic ischemia Curr Surg 1987; 44: 137-139.

10. Sadasivan KK, Carden DL, Moore MB, Korthuis RJ. Neutrophil mediated microvascular injury in acute, experimental compartment syndrome. Clin Orthop Relat Res 1997; 339: 206-215.

11. Liu ZX, Han D, Gunawan B, Kaplowitz N. Neutrophil depletion protects against murine acetaminophen hepatotoxicity. Hepatology 2006; 43: 1220-1230.

12. Ghio AJ, Kennedy TP, Hatch GE, Tepper JS. Reduction of neutrophil influx diminishes lung injury and mortality following phosgene inhalation. J Appl Physiol 1991; 71: 657-665.

13. Apetoh L, Ghiringhelli F, Tesniere A, Obeid M, Ortiz C, Criollo A et al. Toll-like receptor 4-dependent contribution of the immune system to anticancer chemotherapy and radiotherapy. Nat Med 2007; 13: 1050-1059.

14. Casares N, Pequignot MO, Tesniere A, Ghiringhelli F, Roux S, Chaput N et al. Caspasedependent immunogenicity of doxorubicin-induced tumor cell death. J Exp Med 2005; 202: 1691-1701.

15. Matzinger $P$. The danger model: a renewed sense of self. Science 2002; 296: 301-305.

16. Zitvogel L, Kepp O, Kroemer G. Decoding cell death signals in inflammation and immunity. Cell 2010; 140: 798-804

17. Obeid M, Tesniere A, Ghiringhelli F, Fimia GM, Apetoh L, Perfettini JL et al. Calreticulin exposure dictates the immunogenicity of cancer cell death. Nat Med 2007; 13: 54-61.

18. Kono $\mathrm{H}$, Rock KL. How dying cells alert the immune system to danger. Nat Rev Immunol 2008; 8: 279-289. 
19. Hoebe K, Du X, Georgel P, Janssen E, Tabeta K, Kim SO et al. Identification of Lps2 as a key transducer of MyD88-independent TIR signalling. Nature 2003; 424: 743-748.

20. Krysko DV, Vanden Berghe T, D'Herde K, Vandenabeele P. Apoptosis and necrosis: detection, discrimination and phagocytosis. Methods 2008; 44: 205-221.

21. Krysko DV, Vanden Berghe T, Parthoens E, D'Herde K, Vandenabeele P. Methods for distinguishing apoptotic from necrotic cells and measuring their clearance. Methods Enzymol 2008; 442: 307-341.

22. Chen CJ, Shi Y, Hearn A, Fitzgerald K, Golenbock D, Reed G et al. MyD88-dependent IL-1 receptor signaling is essential for gouty inflammation stimulated by monosodium urate crystals. J Clin Invest 2006; 116: 2262-2271.

23. Martinon F, Mayor A, Tschopp J. The inflammasomes: guardians of the body. Annu Rev Immunol 2009; 27: 229-265.

24. Ghiringhelli F, Apetoh L, Tesniere A, Aymeric L, Ma Y, Ortiz $C$ et al. Activation of the NLRP3 inflammasome in dendritic cells induces IL-1beta-dependent adaptive immunity against tumors. Nat Med 2009; 15: 1170-1178.

25. Park JS, Svetkauskaite D, He Q, Kim JY, Strassheim D, Ishizaka A et al. Involvement of Toll-like receptors 2 and 4 in cellular activation by high mobility group box 1 protein. J Biol Chem 2004; 279: 7370-7377.

26. Urbonaviciute V, Furnrohr BG, Meister S, Munoz L, Heyder P, De Marchis F et al Induction of inflammatory and immune responses by HMGB1-nucleosome complexes: implications for the pathogenesis of SLE. J Exp Med 2008; 205: 3007-3018.

27. Tian J, Avalos AM, Mao SY, Chen B, Senthil K, Wu H et al. Toll-like receptor 9-dependent activation by DNA-containing immune complexes is mediated by HMGB1 and RAGE. Nat Immunol 2007; 8: 487-496.

28. Ivanov S, Dragoi AM, Wang X, Dallacosta C, Louten J, Musco G et al. A novel role for HMGB1 in TLR9-mediated inflammatory responses to CpG-DNA. Blood 2007; 110 1970-1981.

29. Kroemer G, Galluzzi L, Vandenabeele P, Abrams J, Alnemri ES, Baehrecke EH et al. Classification of cell death: recommendations of the Nomenclature Committee on Cell Death 2009. Cell Death Differ 2009; 16: 3-11.
30. Krysko DV, D'Herde K, Vandenabeele P. Clearance of apoptotic and necrotic cells and its immunological consequences. Apoptosis 2006; 11: 1709-1726.

31. Bellarosa D, Ciucci A, Bullo A, Nardelli F, Manzini S, Maggi CA et al. Apoptotic events in a human ovarian cancer cell line exposed to anthracyclines. J Pharmacol Exp Ther 2001; 296: 276-283.

32. Tanaka $\mathrm{H}$, Matsushima $\mathrm{H}$, Mizumoto $\mathrm{N}$, Takashima A. Classification of chemotherapeutic agents based on their differential in vitro effects on dendritic cells. Cancer Res 2009; 69: 6978-6986.

33. Chen CJ, Kono H, Golenbock D, Reed G, Akira S, Rock KL. Identification of a key pathway required for the sterile inflammatory response triggered by dying cells. Nat Med 2007; 13: 851-856.

34. Eigenbrod T, Park JH, Harder J, Iwakura Y, Nunez G. Cutting edge: critical role for mesothelial cells in necrosis-induced inflammation through the recognition of IL-1 alpha released from dying cells. J Immunol 2008; 181: 8194-8198.

35. Cavassani KA, Ishii M, Wen H, Schaller MA, Lincoln PM, Lukacs NW et al. TLR3 is an endogenous sensor of tissue necrosis during acute inflammatory events. J Exp Med 2008; 205: 2609-2621.

36. Garg AD, Nowis D, Golab J, Vandenabeele P, Krysko DV, Agostinis P. Immunogenic cell death, DAMPs and anticancer therapeutics: an emerging amalgamation. Biochim Biophys Acta 2010; 1805: 53-71.

37. Chase MA, Wheeler DS, Lierl KM, Hughes VS, Wong HR, Page K. Hsp72 induces inflammation and regulates cytokine production in airway epithelium through a TLR4- and NF-kappaB-dependent mechanism. J Immunol 2007; 179: 6318-6324.

38. Wheeler DS, Chase MA, Senft AP, Poynter SE, Wong HR, Page K. Extracellular Hsp72, an endogenous DAMP, is released by virally infected airway epithelial cells and activates neutrophils via Toll-like receptor (TLR)-4. Respir Res 2009; 10: 31.

39. Scaffidi $P$, Misteli T, Bianchi ME. Release of chromatin protein HMGB1 by necrotic cells triggers inflammation. Nature 2002; 418: 191-195.

40. Dasari P, Nicholson IC, Hodge G, Dandie GW, Zola H. Expression of Toll-like receptors on B lymphocytes. Cell Immunol 2005; 236: 140-145. 